\title{
There are no Quantum Jumps, nor are there Particles!
}

\author{
H. D. Zeh \\ Institut für Theoretische Physik \\ Universität Heidelberg
}

\begin{abstract}
Quantum theory does not require the existence of discontinuities: neither in time (quantum jumps), nor in space (particles), nor in spacetime (quantum events). These apparent discontinuities are readily described objectively by the continuous process of decoherence occurring locally on a very short time scale according to the Schrödinger equation for interacting systems, while the observer's 'increase of information' is appropriately represented by the resulting dynamical decoupling of the corresponding components of the global wave function.
\end{abstract}

Physics Letters A172, 189 (1993) 
As far as is known, all properties of closed quantum systems are perfectly described by means of wave functions in configuration space (in general, wave functionals of certain fields) dynamically evolving smoothly according to the time-dependent Schrödinger equation. However, the condition of being closed (or shielded against interactions with the environment) can easily be estimated to be quite exceptional. It characterizes very special (usually atomic) systems from which the laws of quantum mechanics were derived. When the shielding ceases, most notably during measurements, discontinuous events ('quantum jumps' or a 'collapse of the wave function') seem to occur, and particle aspects seem to be observed. Such events are also known to lead to a loss of interference between different values of the 'measured' variables - regardless of whether any result is read from the apparatus by an observer.

Macroscopic systems are very effectively coupled to their environment in this way. They cannot avoid being 'continuously measured' in the sense of losing interference. ${ }^{1}$ This is obvious without any calculation, since we could never see macroscopic objects if they did not continuously scatter light which thereby had to carry away 'information' about their position and shape. The effect of such interactions is often taken into account dynamically by means of stochastic terms in the evolution of the wave function of the considered system (sometimes called 'chopping' or 'kicking') - equivalent to a nonunitary evolution of the density matrix. ${ }^{2}$ These terms (introduced ad hoc) are usually interpreted as representing fundamental aspects of quantum mechanics (just as the supplementary dynamics that von Neumann introduced as his 'first intervention' to augment the Schrödinger equation in the case of measurements $\operatorname{proper}^{3}$ ).

Precisely such empirically justified dynamical terms can however be derived within the well established quantum mechanics of interacting systems provided the environment is properly taken into account. ${ }^{4}$ (For a recent review of this approach see Zurek. ${ }^{5}$ ) Joos and $\mathrm{Zeh}^{6}$ have calculated that even small dust particles or large molecules must 'decohere' (that is, lose certain interference terms) on a time scale of fractions of a second, while Zurek $^{7}$ has estimated that for a normal macroscopic system the rate of decoherence is typically faster than thermal relaxation by an astounding factor of the order of $10^{40}$. In contrast, microscopic systems tend to decohere into energy eigenstates, since they interact with their environment mainly through their decay products. It is for this reason that the time-independent Schrödinger equation is so useful for describing atomic systems. In quantum measurements proper, microscopic properties will first become correlated with macroscopically different pointer positions, the superpositions of which must then immediately decohere in the described way. 
For all practical purposes, this short time scale of decoherence can be interpreted as the occurrence of dynamical discontinuities. In laser physics, shielding from the environment can be controlled by the experimenter to a great extent. Even single atoms can be brought into a position of having their decay status 'continuously measured', so that quantum jumps seem to be directly visible, ${ }^{8}$ while in the opposite situation of sufficiently closed systems (single atoms in a cavity) the superposition of different decay times may become important and lead to coherent revival of the initial decaying state. ${ }^{9}$

It turns out furthermore that decoherence preferentially destroys interference between those parts of the wave function which differ markedly in position. This leads to density matrices which are effectively equivalent to ensembles of narrow wave packets. Such wave packets may then be interpreted as representing individual 'particle' positions (i.e., no ensembles of positions any more), precisely as originally envisioned by Schrödinger. ${ }^{10}$ Their spreading with time according to the Schrödinger equation is counteracted by continuing decoherence, resulting in a certain (observable) indeterminism of their quasi-classical orbits ('quantum Brownian motion') even if recoil is neglected. ${ }^{6}$ All particle aspects observed in measurements of quantum fields (like spots on a plate, tracks in a bubble chamber, or clicks of a counter) can be understood by taking into account this decoherence of the relevant local (i.e., subsystem) density matrix. (The concept of 'particle numbers' is of course explained by the oscillator quantum numbers of the corresponding field modes - at least for bosons.)

In fact, all classical aspects (or the apparent validity of fundamental superselection rules) seem to be derivable in this way from the assumption of a global Schrödinger wave function(al). It is the unavoidable environment that determines which properties decohere (that is, become classical). Charge eigenstates, for example, decohere locally by being 'kinematically' quantum-correlated with the surrounding electromagnetic field state as required by Gauss' law in the form of the conserved constraint [divE $\mathbf{E}-4 \pi \rho] \Psi=0$. When pairs of charges are created (and spatially separated), decoherence of the individual charges by their fields becomes a dynamical process again (equivalent to an irreversible measurement-like process once photons registrating the resulting dipole and higher moments are emitted or scattered).

I do not know of any apparent violation of the Schrödinger equation or the superposition principle that cannot at least plausibly be expected to be derivable in terms of decoherence. In spite of this success (which can hardly be an accident), this description is often considered as insufficient to explain the measurement process itself. ${ }^{41}$ The reservations do seem sound, since decoherence is described formally by means of the density matrix of the considered subsystem of the universe, obtained by tracing out the 
rest (the 'environment'). The concept of the density matrix (of subsystems in this case) is however justified itself only as a means for calculating expectation values or probabilities for outcomes of further measurements, that is, for the secondary quantum jumps which would have to occur, for example, when the pointer is read. This explanation of measurements therefore seems to be circular from a fundamental point of view. In the global wave function (which is interpreted as representing 'reality' in this picture) all interference terms remain present. The universe as a whole never decoheres. The description of measurements by means of merely local decoherence - so goes the usual argument - must be wrong, since one does observe, in contrast to this global superposition of different outcomes derived from the Schrödinger equation, that only one of its components (a wave packet representing a definite outcome) exists after every measurement.

However, this latter claim is wrong, and so is the argument. For after an observation one need not necessarily conclude that only one component now exists but only that only one component is observed. But this fact is readily described by the Schrödinger equation without any modification. Whenever an observer interacts with the measurement device in a way that corresponds to an observation of the result, his own state must be quantumcorrelated with the macroscopic pointer position (and potentially also with other observers), and hence be decohered from the beginning. Superposed world components describing the registration of different macroscopic properties by the 'same' observer are dynamically entirely independent of one another: they describe different observers. Because of the fork-like structure of causality (the spreading in space of the retarded effects of local causes) ${ }^{12}$, there is no chance of their forming a superposition with respect to (or in) a local observer any more (except, perhaps, in a recollapsing Friedmann universe).

This dynamical consequence of decoherence explains everything that has to be explained dynamically in order to understand what can be observed by local observers. He who considers this conclusion of an indeterminism or splitting of the observer's identity, derived from the Schrödinger equation in the form of dynamically decoupling ('branching') wave packets on a fundamental global configuration space, as unacceptable or "extravagant"13 may instead dynamically formalize the superfluous hypothesis of a disappearance of the 'other' components by whatever method he prefers, but he should be aware that he may thereby also create his own problems: Any deviation from the global Schrödinger equation must in principle lead to observable effects, and it should be recalled that none have ever been discovered. ${ }^{14}$ The conclusion would of course have to be revised if such effects were some day to be found. But as of now, there is no objective reason to expect them to exist; and even if they did, they need not take the form of the 
apparent discontinuities which are readily described by means of local decoherence according to the universal Schrödinger equation.

This is not to deny the existence of several open problems. In particular, (1) the fundamental 'arena' of wave mechanics, which may or may not correspond to a classical configuration space, can only be known once we possess a fundamental 'Theory of Everything'. Quantum gravity indicates that this arena must have an interesting metric structure ('superspace') which leads to a stationary Schrödinger equation of hyperbolic nature (the Wheeler-DeWitt equation). ${ }^{15}$ (2) The precise definition of the dynamically independent components ('branches') remains elusive. ${ }^{16}$ However, this specification is unimportant for understanding the objective part of measurements (in particular, decoherence), just as a detailed theory of conscious observers was not required for describing measurements in a classical world. Hence, in contrast to dynamical collapse models proposed in the literature, ${ }^{17}$ this branching does not correspond to the apparently observed quantum jumps. It only justifies their description by means of decoherence of the local density matrix, provided the usual locality of interactions remains valid. (In the orthodox interpretation, decoherence would still occur as an objective process in the measurement device even if the 'Heisenberg cut' were shifted deep into the observer.) (3) In contrast to an assertion by Everett, the law of quantum probabilities cannot be derived without further (though weak) assumptions about the selection of 'our' world component. ${ }^{18}(4)$ The most important underivable assumption in a kinematically nonlocal (i.e., nonseparable) quantum world seems to be the locality of the ultimate (subjective) observer in spacetime (required in some vague but essential form). Quantum decoherence is meaningful (or 'relevant') only with respect to local parts of the nonlocal quantum world.

None of these open problems indicates an inconsistence or limitation of the description of the whole world by means of the Schrödinger equation - only a lack of knowledge about important details (mainly about the quantum physical description of an observer). Hence, there does not seem to be any reasonable motivation (other than traditionalism) for introducing concepts like particles, quantum jumps, superselection rules, or classical properties on a fundamental level. It also appears unfortunate, therefore, that the now very popular technical concept of path integrals may suggest a fundamental role of paths or 'histories' of classical states, although their required superposition is nothing but another representation of the Schrödinger wave function. (Feynman's method could similarly be applied for computing the propagation of classical waves by sums over paths in three-dimensional space, without giving these paths any physical meaning.) Quasiclassical histories ${ }^{19}$ emerge instead from the global wave function as traveling and thereby smoothly branching narrow wave packets (not as time sequences of 'events') 
only because of the continuous action of decoherence (which leads to increasing complexity, symmetry-breaking, and fine-graining into dynamically independent wave packets). This statement applies also to spacetime with its metric structure that must be part of the quasiclassical history. ${ }^{20}$ It would be insufficient simply to require decoherence just because it leads to classically plausible consequences (such as 'consistent histories').

It thus appears becoming evident that our classical concepts describe mere shadows on the wall of Plato's cave in which we are living. Using them for describing reality must lead to 'paradoxes'.

Acknowledgment: I wish to thank Julian Barbour, Erich Joos and Claus Kiefer for discussions and for suggesting a number of helpful changes in the manuscript.

\section{References}

${ }^{1}$ H.D. Zeh, Found.Phys. 1 (1970) 69; H.D. Zeh, Enrico Fermi School of Physics IL (Academic Press, 1971) 263; O. Kübler and H.D. Zeh, Ann. Phys. (N.Y.) 76 (1973) 405; W.H. Zurek, Phys. Rev. D24 (1981) 1516; W.H. Zurek, Phys. Rev. D26 (1982) 1862.

${ }^{2}$ I do not feel able to give anything like a complete account of the numerous contributions of this kind. This Letter was written in response to N. Gisin and I. C. Percival, Phys. Lett. A167 (1992) 315.

3 J. von Neumann, Mathematische Grundlagen der Quantenmechanik (Springer, 1932); Engl. transl.: Mathematical Foundations of Quantum Mechanics (Princeton Univ. Press, 1955), Ch. V.1.

${ }^{4}$ E. Joos, Phys. Rev. D36 (1987) 3285; M.R. Gallis and G.N. Fleming, Phys. Rev. A43 (1991) 5778.

5 W.H. Zurek, Phys. Today 44 (Oct. 1991) 36; for more recent contributions see E. Calzetta, M. Castagnino, and R. Scoccimarro, Phys. Rev. D45 (1992) 2806; B.L. Hu, J.P. Paz, and Y. Zhang, Phys. Rev. D45 (1992) 2843; C. Kiefer, Phys. Rev. D46 (1992) 1658.

${ }^{6}$ E. Joos and H.D. Zeh, Z. Phys. B59 (1985) 223.

${ }^{7}$ W.H. Zurek, in: Frontiers of Nonequilibrium Statistical Physics, edts. G.T. Moore and E.T. Sculley (Plenum, 1986).

${ }^{8}$ Th. Sauter, W. Neuhauser, R. Blatt and P.E. Toschek, Phys. Rev. Lett. 57 (1986) 1696.

${ }^{9}$ G. Rempe, H. Walther and N. Klein, Phys. Rev. Lett. 58 (1987) 353.

${ }^{10}$ E. Schrödinger, Z. Physik 14 (1926) 664.

${ }^{11}$ G. C. Ghirardi, A. Rimini, and T. Weber, Phys. Rev. D36 (1987) 3287.

${ }^{12}$ H.D. Zeh, The Physical Basis of the Direction of Time, 2nd edition, (Springer, 1992).

${ }^{13}$ J.S. Bell, in: Quantum Gravity II, edts. C.J. Isham, R. Penrose and D.W. Sciama (Clarendon Press, 1981). 
${ }^{14}$ S. Weinberg, Phys. Rev. Lett. 62 (1989) 485; J. Polchinski, Phys. Rev. Lett. 66 (1991) 397; E.J. Squires, Phys. Lett. A163 (1992) 356.

${ }^{15}$ B.S. DeWitt, Phys. Rev. 160 (1967) 1113.

${ }^{16}$ H.D. Zeh, Found. Phys. 3 (1973) 109; H.D. Zeh, Found. Phys. 9 (1979) 803; A.O. Barvinsky and A.Yu. Kamenshchik, Class. Qu. Gravity 7 (1990) 2285; A. Albrecht, Identifying decohering paths in closed quantum systems (Fermi-Lab preprint, 1990).

${ }^{17}$ G. C. Ghirardi, A. Rimini, and T. Weber, Phys. Rev. D34 (1986) 470; L. Diósi, Phys. Lett. A120 (1987) 377.

18 J.B. Hartle, Am. J. Phys. 36 (1968) 704; N. Graham: The Everett interpretation of quantum mechanics, Thesis (Univ. North Carolina, Chapel Hill, 1970).

19 A.O. Caldeira and A.J. Leggett, Physica 121A (1983) 587; R.B. Griffiths, J. Stat. Phys. 36 (1984) 219; M. Gell-Mann and J.B. Hartle, in: Complexity, Entropy and the Physics of Information, edt. W.H. Zurek, (Addison-Wesley, 1990); R. Omnès, Rev. Mod. Phys. 64 (1992) 339.

${ }^{20}$ E. Joos, Phys. Lett. A116 (1986) 6; H.D. Zeh, Phys. Lett. A116 (1986) 9; C. Kiefer, Class. Qu. Gravity 4 (1987) 1369. 